\title{
Glycosides of Atractylodes ovata
}

\author{
Junichi Kitajima, ${ }^{*, a}$ Akane Kamoshita, ${ }^{a}$ Toru Ishikawa, ${ }^{a}$ Akihito Takano, ${ }^{a}$ Tatsuo FukudA, ${ }^{b}$ \\ Susumu IsODA, ${ }^{c}$ and Yoshiteru IDA ${ }^{c}$ \\ ${ }^{a}$ Showa Pharmaceutical University; 3 Higashi-Tamagawagakuen, Machida, Tokyo 194-8543, Japan: ${ }^{b}$ Tokyo Metropolitan \\ Medical Plants Garden; 21-1 Nakajima-machi, Kodaira, Tokyo 187-0033, Japan: and ${ }^{c}$ Showa University School of \\ Pharmaceutical Sciences; Hatanodai, Shinagawa-ku, Tokyo 142-8555, Japan.
}

Received May 9, 2003; accepted June 20, 2003

\begin{abstract}
A new coumarin glycoside and a new glycoside of an acetylene derivative were isolated from the water-soluble portion of the methanolic extract of Atractylodes ovata rhizome together with eight known compounds. Their structures were characterized as scopoletin $\beta$-D-xylopyranosyl-(1 $\rightarrow 6)$ - $\beta$-D-glucopyranoside and $(2 E)-2$-decene4,6-diyne-1,8-diol 8-O- $\beta$-D-apiofuranosyl-(1 $\rightarrow 6)$ - $\beta$-D-glucopyranoside, respectively, based on chemical and spectroscopic investigations. A comparison of the polar constituents among Atractylodes japonica, Atractylodes lancea, and $A$. ovata is led to the conclusion that $A$. ovata is distinguishable from $A$. lancea and $A$. japonica, as also shown by phylogenetic analysis.
\end{abstract}

Key words Atractylodes ovata; Atractylodes Rhizome; chemotaxonomy; scopoletin glycoside; 2-decene-4,6-diyne-1,8-diol glycoside

In the previous paper, we reported the isolation and characterization of eight sesquiterpenoid glycosides, including atractyloside $\mathrm{A}-\mathrm{E}, \mathrm{G}$, and a secoguaiane derivative, a monoterpenoid glucoside, seven aromatic compound glycosides, and L-phenylalanine from the water-soluble portion of the rhizome of Atractylodes japonica. ${ }^{1)}$ In addition, 16 sesquiterpenoid glycosides, four monoterpenoid glucosides, two hemiterpenoid glycosides, an alkyl glycoside, five aromatic compound glycosides, an acetylene derivative compound glucoside, two nucleosides, and L-tryptophan were isolated from the water-soluble portion of the rhizome of Atractylodes lancea. ${ }^{2,3)}$ The rhizomes of Atractylodes plants are classified into two groups that contain $\beta$-eudesmol and hinesol as the main constituents of the essential oil ( $A$. lancea and Atractylodes chinensis; so-jutsu), and atractylon as the main constituent of the essential oil (A. japonica and Atractylodes ovata; byaku-jutsu) in the Japanese Pharmacopoeia. ${ }^{4)}$ Sometimes, however, the rhizomes are prescribed in traditional medicine indistinguishably. In addition, $A$. ovata is distinguishable from other Atractylodes plants using RAPD analysis, ${ }^{5)}$ and the phylogenetic relationship between $A$. japonica and $A$. lancea is suggested to be closer than that between $A$. japonica and $A$. ovata. We then undertook examination of the water-soluble portion of $A$. ovata to determine the chemotaxonomic relationships among $A$. ovata, A. japonica, and A. lancea.

The dried rhizome of $A$. ovata, which was cultivated in the Tokyo Metropolitan Medical Plants Garden, was extracted with $70 \%$ methanol, and the methanolic extract was suspended in water and successively extracted with ether and ethyl acetate. The aqueous layer was chromatographed on Amberlite XAD-II to give water and methanol eluate fractions. The methanol eluate fraction was chromatographed on Sephadex LH-20 followed by silica gel, Lobar RP-8 column chromatography, and HPLC, affording a coumarin glycoside (1), an acetylene derivative compound glycoside (2), four aromatic compound glycosides $(\mathbf{3}-\mathbf{6})$, three guaiane-type sesquiterpenoid glucosides (7-9), and L-tryptophan (10). Among them, $\mathbf{1}$ and $\mathbf{2}$ are new, and their structures were characterized as follows. Their molecular formulae were sug- gested from the accurate mass number of the $[\mathrm{M}+\mathrm{H}]^{+}$ion peak in the high-resolution positive FAB-MS.

The major coumarin glycoside $\mathbf{1}, \mathrm{C}_{21} \mathrm{H}_{26} \mathrm{O}_{13}$, showed $[\mathrm{M}+\mathrm{K}]^{+}$and $[\mathrm{M}+\mathrm{H}]^{+}$ion peaks at $\mathrm{m} / z 525$ and 487 , respectively, in the positive FAB-MS. Enzymatic hydrolysis of $\mathbf{1}$ gave an aglycone (1a), which was identified as scopoletin (7hydroxy-6-methoxycoumarin), and D-glucose and D-xylose as the sugar components. The ${ }^{13} \mathrm{C}-\mathrm{NMR}$ data of $\mathbf{1}$ were similar to those of scopoletin $\beta$-D-glucopyranoside (1b), ${ }^{6}$ except for the signals due to a $\beta$-D-xylopyranosyl group. Cross-peaks between the C-7/glucosyl H-1 and glucosyl C-6/xylosyl H-1 were observed in the heteronuclear multiple bond connectivity (HMBC) spectrum of $\mathbf{1}$ (see Experimental), suggesting that the xylosyl group was located at C- 6 of the glucose in 1b. This was also supported by the cross-peaks observed between $\mathrm{H}-5 /-\mathrm{OCH}_{3}$ and between $\mathrm{H}-8 /$ glucosyl H-1 in its nuclear Overhauser and exchange spectroscopy (NOESY) spectrum. Therefore 1 was characterized as scopoletin $\beta$-D-xylopyranosyl- $(1 \rightarrow 6)$ - $\beta$-D-glucopyranoside.

Acetylene derivative compound glycoside $2, \mathrm{C}_{21} \mathrm{H}_{30} \mathrm{O}_{11}$, showed $[\mathrm{M}+\mathrm{H}]^{+}$and $\left[\mathrm{M}-\mathrm{C}_{11} \mathrm{H}_{20} \mathrm{O}_{10}+\mathrm{H}\right]^{+}$ion peaks at $\mathrm{m} / \mathrm{z}$ 459 and 183, respectively, in the positive FAB-MS, and $[\mathrm{M}-\mathrm{H}]^{-}$and $\left[\mathrm{M}-\mathrm{C}_{11} \mathrm{H}_{20} \mathrm{O}_{10}-\mathrm{H}\right]^{-}$ion peaks at $\mathrm{m} / z 457$ and 181, respectively, in the negative FAB-MS. The NMR data showed the presence of two triple bonds, one disubstituted double bond, one prim-methyl, one hydroxymethyl, one methylene, and one oxygenated methine in addition to the $\beta$ D-apiofuranosyl-( $1 \rightarrow 6)$ - $\beta$-D-glucopyranosyl group. ${ }^{1)}$ Comparison of its NMR data with those of $(2 E, 8 E)$-decadiene4,6-diyne-1,10-diol 1-O- $\beta$-D-glucopyranoside, which was isolated from the rhizome of A. lancea ${ }^{3)}$ and the results of an HMBC experiment (Fig. 1) showed that the aglycone of $\mathbf{2}$ was 2-decene-4,6-diyne-1,8-diol, and the glycosyl group was located at C-8. Therefore 2 was concluded to be $(2 E)$ decene-4,6-diyne-1,8-diol 8 - $O$ - $\beta$-D-apiofuranosyl- $(1 \rightarrow 6)-\beta$ D-glucopyranoside.

Aromatic compound glycosides $\mathbf{3}$ to $\mathbf{6}$ and guaiane-type sesquiterpenoid glucosides $\mathbf{7}$ to $\mathbf{9}$ were identified as icariside $\mathrm{F}_{2},{ }^{7}$ icariside $\mathrm{D}_{1},{ }^{7}$ syringin, ${ }^{7}$ dihydrosyrindine, ${ }^{8)}$ atractyloside $\mathrm{A},{ }^{2}{ }^{2}$ 10-epi-atractyloside $\mathrm{A},{ }^{3)}$ and atractyloside $\mathrm{B},{ }^{2)}$ re- 
spectively (Fig. 2).

While the commom guaiane-type sesquiterpenoid glucosides were found in the rhizomes of $A$. japonica, A. lancea, and $A$. ovata, we were able to isolate the new characteristic coumarin glycoside $\mathbf{1}$ as the main glycoside of the rhizome of $A$. ovata. A comparison of the polar constituents among $A$. japonica, A. lancea, and $A$. ovata (Table 1) showed that $A$. ovata is distinguishable from A. lancea and A. japonica, as did the phylogenetic analysis.

\section{Experimental}

The instruments used and the experimental conditions for spectral mesurements and chromatography were the same as those reported in the previous papers. ${ }^{1,3)}$ Symmetryprep C18 $7 \mu \mathrm{m}$ (Waters; column size, $7.8 \times$ $300 \mathrm{~mm}$; ODS) and Carbohydrate analysis (Waters; column size, $3.9 \times$ $300 \mathrm{~mm}$; CHA) were used as columns for HPLC separations.

Extraction and Separation The dried rhizome of A. ovata $(1.5 \mathrm{~kg})$, which was cultivated in the Tokyo Metropolitan Medical Plant Garden (Kodaira, Tokyo, Japan), was extracted with $70 \%$ methanol $(51 \times 3)$ for 2 weeks, and the extract $(573.5 \mathrm{~g})$ was partitioned between ether/water and then ethyl acetate/water. The aqueous portion $(555.3 \mathrm{~g})$ was chromatographed over Amberlite XAD-II $\left(\mathrm{H}_{2} \mathrm{O} \rightarrow \mathrm{MeOH}\right)$ to give a water eluate $(521.0 \mathrm{~g})$ and a methanol eluate $(34.3 \mathrm{~g})$.

The methanol eluate was subjected to Sephadex $\mathrm{LH}-20\left[\mathrm{MeOH}-\mathrm{H}_{2} \mathrm{O}\right.$ $(9: 1)]$ to give six fractions (frs. A-F). Fraction $\mathrm{C}(14.72 \mathrm{~g})$ was chromatographed on silica gel $\left[\mathrm{CHCl}_{3}-\mathrm{MeOH}-\mathrm{H}_{2} \mathrm{O}(4: 1: 0.1 \rightarrow 7: 3: 0.5 \rightarrow\right.$ $6: 4: 0.5) \rightarrow \mathrm{MeOH}]$ to give 15 fractions (frs. $\left.\mathrm{C}_{1}-\mathrm{C}_{15}\right)$. Fraction $\mathrm{C}_{4}(0.13 \mathrm{~g}$ ) was passed through a Lobar RP-8 column $\left[\mathrm{MeCN}-\mathrm{H}_{2} \mathrm{O}(3: 17)\right]$ to give 11 fractions (frs. $\mathrm{C}_{4-1}-\mathrm{C}_{4-11}$ ), and fr. $\mathrm{C}_{4-3}$ was subjected to HPLC [ODS, $\left.\mathrm{MeCN}-\mathrm{H}_{2} \mathrm{O}(3: 17)\right]$ to give $5(6 \mathrm{mg})$ and $6(5 \mathrm{mg})$. Fraction $\mathrm{C}_{6}(0.13 \mathrm{~g})$ was passed through a Lobar RP-8 column $\left[\mathrm{MeCN}-\mathrm{H}_{2} \mathrm{O}(3: 17)\right]$ to give 11 fractions (frs. $\mathrm{C}_{6-1}-\mathrm{C}_{6-11}$ ), fr. $\mathrm{C}_{6-7}$ was subjected to HPLC [CHA, MeCN- $\mathrm{H}_{2} \mathrm{O}$

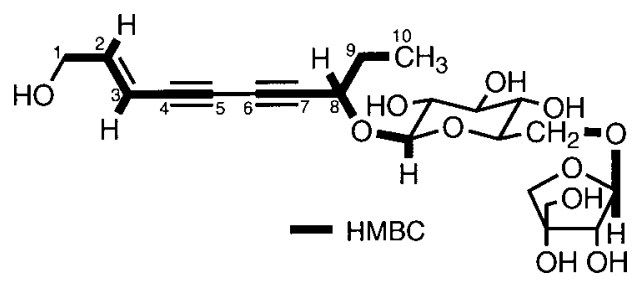

(19:1)] to give $3(65 \mathrm{mg})$, and fr. $\mathrm{C}_{6-10}$ was subjected to HPLC [ODS, $\left.\mathrm{MeCN}-\mathrm{H}_{2} \mathrm{O}(3: 17)\right]$ to give $4(20 \mathrm{mg})$. Fraction $\mathrm{C}_{7}(0.50 \mathrm{~g})$ was passed through a Lobar RP-8 column $\left[\mathrm{MeCN}-\mathrm{H}_{2} \mathrm{O}(3: 17)\right]$ to give nine fractions (frs. $\mathrm{C}_{7-1}-\mathrm{C}_{7-9}$ ). Fraction $\mathrm{C}_{7-4}$ was recrystallized from methanol to give $\mathbf{1}$ $(200 \mathrm{mg})$, and fr. $\mathrm{C}_{7-8}$ was subjected to HPLC [ODS, MeCN- $\mathrm{H}_{2} \mathrm{O}(3: 17)$ and $\left.\mathrm{CHA}, \mathrm{MeCN}-\mathrm{H}_{2} \mathrm{O}(24: 1)\right]$ to give $2(2 \mathrm{mg})$. Fraction $\mathrm{C}_{9}(0.33 \mathrm{~g})$ was passed through a Lobar RP-8 column $\left[\mathrm{MeCN}-\mathrm{H}_{2} \mathrm{O}(3: 17)\right]$ to give seven fractions (frs. $\mathrm{C}_{9-1}-\mathrm{C}_{9-7}$ ), and fr. $\mathrm{C}_{9-3}$ was subjected to HPLC [ODS, $\left.\mathrm{MeCN}-\mathrm{H}_{2} \mathrm{O}(3: 37)\right]$ to give $8(7 \mathrm{mg})$ and $7(40 \mathrm{mg})$. Fraction $\mathrm{C}_{11}(1.31 \mathrm{~g})$ was passed through a Lobar RP-8 column $\left[\mathrm{MeCN}-\mathrm{H}_{2} \mathrm{O}(3: 17)\right]$ to give seven fractions (frs. $\mathrm{C}_{11-1}-\mathrm{C}_{11-7}$ ). Fraction $\mathrm{C}_{11-3}$ was subjected to HPLC [CHA, MeCN- $\left.\mathrm{H}_{2} \mathrm{O}(14: 1)\right]$ to give $9(16 \mathrm{mg})$, and fr. $\mathrm{C}_{11-4}$ was subjected to Sephadex LH-20 (MeOH) to give $\mathbf{1 0}(15 \mathrm{mg})$.

The following compounds were identified by comparison with authentic compounds or published physical and spectral data: icariside $F_{2}(3)$, icariside $\mathrm{D}_{1}$ (4), syringin (5), dihydrosyrindine (6), atractyloside $\mathrm{A}$ (7), 10-epiatractyloside A (8), atractyloside B (9), and L-tryptophan (10).

Scopoletin 7- $O$ - $\beta$-D-Xylopyranosyl- $(1 \rightarrow 6)-\beta$-D-glucopyranoside $(\mathbf{1})$ : Colorless needles $(\mathrm{MeOH}), \mathrm{mp} 243-245^{\circ} \mathrm{C},[\alpha]_{\mathrm{D}}^{23}-148^{\circ}\left(c=0.5, \mathrm{H}_{2} \mathrm{O}\right)$. Positive FAB-MS $m / z$ : $525.1011[\mathrm{M}+\mathrm{K}]^{+}$(Calcd for $\mathrm{C}_{21} \mathrm{H}_{26} \mathrm{KO}_{13}, 525.1011$ ), $487.1465[\mathrm{M}+\mathrm{H}]^{+}$(base, Calcd for $\mathrm{C}_{21} \mathrm{H}_{27} \mathrm{O}_{13}, 487.1452$ ). ${ }^{1} \mathrm{H}-\mathrm{NMR}$ $\left(\mathrm{DMSO}-d_{6}, 500 \mathrm{MHz}\right) \delta: 6.33(1 \mathrm{H}, \mathrm{d}, J=9.5 \mathrm{~Hz}, \mathrm{H}-3), 7.96(1 \mathrm{H}, \mathrm{d}$, $J=9.5 \mathrm{~Hz}, \mathrm{H}-4), 7.30$ (1H, s, H-5), 7.20 (1H. s. H-8), $3.82\left(3 \mathrm{H}, \mathrm{s}, 6-\mathrm{OCH}_{3}\right)$, $5.10\left(1 \mathrm{H}, \mathrm{d}, J=7.5 \mathrm{~Hz}\right.$, Glc H-1), $4.12(1 \mathrm{H}, \mathrm{d}, J=7.5 \mathrm{~Hz}, \mathrm{Xyl} \mathrm{H}-1) .{ }^{13} \mathrm{C}-$ NMR (DMSO- $\left.d_{6}, 125 \mathrm{MHz}\right) \delta$ : 160.50 (C-2), 113.34 (C-3), 144.14 (C-4), 109.72 (C-5), 145.96 (C-6), 149.76 (C-7), 103.01 (C-8), 148.92 (C-9), 112.31 (C-10), $56.01\left(6-\mathrm{OCH}_{3}\right), 99.50$ (Glc C-1), 72.99 (Glc C-2), 76.52 (Glc C-3), 69.19 (Glc C-4), 75.33 (Glc C-5), 68.17 (Glc C-6), 104.02 (Xyl C-1), 73.25 (Xyl C-2), 76.54 (Xyl C-3), 69.42 (Xyl C-4), 65.60 (Xyl C-5). HMBC correlations: H-3/C-2, C-10; H-4/C-2, C-5, C-9, C-10; H-5/C-4, C6, C-7, C-9, C-10; H-8/C-6, C-7, C-9, C-10; $-\mathrm{OCH}_{3} / \mathrm{C}-6$; Glc H-1/C-7; Xyl H-1/Glc C-6.

Enzymatic Hydrolysis of 1 A mixture of $1(25 \mathrm{mg})$ and $\beta$-glucosidase ( $5 \mathrm{mg}$; Toyobo Co. Ltd., Lot 93240) in water $(5 \mathrm{ml})$ was shaken in a water bath at $37^{\circ} \mathrm{C}$ for $5 \mathrm{~d}$. The mixture was concentrated in vacuo to dryness and the residue was chromatographed over silica gel $\left[\mathrm{CHCl}_{3}-\mathrm{MeOH}(4: 1\right.$ to $1: 1)$ ] to afford an aglycone $(\mathbf{1 a} ; 10 \mathrm{mg})$ and a sugar fraction. The sugar fraction was passed through Sephadex LH-20 (MeOH) to give a syrup, and HPLC [carbohydrate analysis (Waters); detector, JASCO RI-930 detector and JASCO OR-990 chiral detector; solv., $\mathrm{MeCN}-\mathrm{H}_{2} \mathrm{O}(9: 1), 2 \mathrm{ml} / \mathrm{min} ; t_{\mathrm{R}}$ $4.20 \mathrm{~min}$ (same location as that of $\mathrm{D}$-xylose) and $t_{\mathrm{R}} 6.80 \mathrm{~min}$ (same location as that of D-glucose)] showed the presence of D-glucose and D-xylose.

Scopoletin (1a): Colorless needles (EtOH), mp 205-207 ${ }^{\circ} \mathrm{C},{ }^{1} \mathrm{H}-\mathrm{NMR}$ $\left(\mathrm{DMSO}-d_{6}, 270 \mathrm{MHz}\right) \delta: 6.21(1 \mathrm{H}, \mathrm{d}, J=9.5 \mathrm{~Hz}, \mathrm{H}-3), 7.91 \quad(1 \mathrm{H}, \mathrm{d}$, $J=9.5 \mathrm{~Hz}, \mathrm{H}-4), 7.21$ (1H, s, H-5), 6.77 (1H. s. H-8), $3.81\left(3 \mathrm{H}, \mathrm{s}, 6-\mathrm{OCH}_{3}\right)$.

Fig. 1. Structure and HMBC Correlations of 2

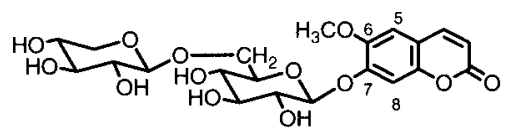

1
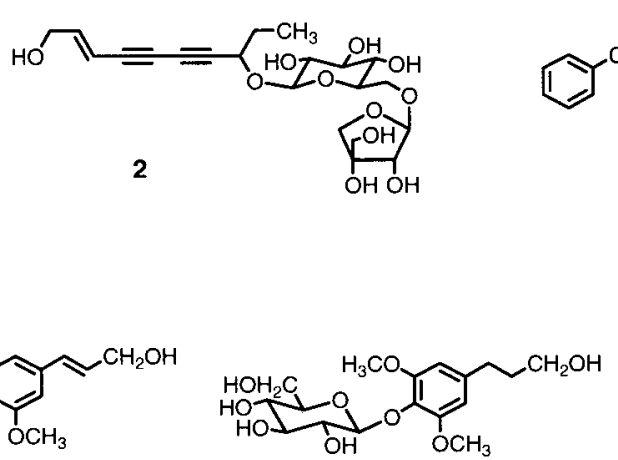

6
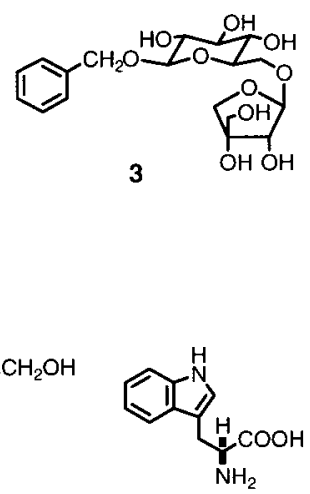

10

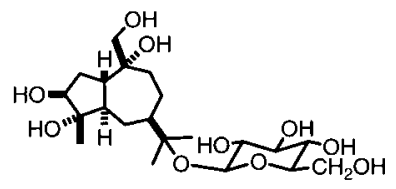

9

Fig. 2. Structures of $\mathbf{1}-\mathbf{1 0}$ 
Table 1. Polar Constituents of A. japonica, A. lancea, and A. ovata Rhizome

\begin{tabular}{|c|c|c|c|c|}
\hline & Type & A. japonica & A. lancea & A. ovata \\
\hline \multicolumn{5}{|c|}{ Sesquiterpenoid alcohol glycosides } \\
\hline \multirow{7}{*}{ Guaiane-type } & 7 & +++++ & +++++ & +++ \\
\hline & 8 & ++ & +++ & + \\
\hline & 9 & ++ & ++ & ++ \\
\hline & 11 & +++ & +++++ & \\
\hline & 12 & + & +++ & \\
\hline & 14 & & +++ & \\
\hline & 15 & & ++ & \\
\hline Secoatractylolactone-type & 13 & ++ & +++ & \\
\hline \multirow[t]{9}{*}{ Eudesmane-type } & 35 & +++ & & \\
\hline & 16 & & ++++ & \\
\hline & 17 & & ++++ & \\
\hline & 18 & & + & \\
\hline & 19 & & ++ & \\
\hline & 20 & & ++ & \\
\hline & 21 & & + & \\
\hline & 22 & & +++ & \\
\hline & 23 & & +++ & \\
\hline \multicolumn{5}{|c|}{ Hemiterpenoid alcohol glycosides } \\
\hline & 28 & & + & \\
\hline & 29 & & + & \\
\hline \multicolumn{5}{|c|}{$\underline{\text { Monoterpenoid alcohol glucosides }}$} \\
\hline \multirow[t]{2}{*}{ 1,8-Cineole-type } & 24 & + & + & \\
\hline & 25 & & + & \\
\hline \multirow[t]{2}{*}{$p$-Menthane-type } & 26 & & + & \\
\hline & 27 & & + & \\
\hline \multicolumn{5}{|l|}{ Aromatic glycosides } \\
\hline \multirow[t]{4}{*}{$\mathrm{C}_{6}$ type } & 31 & + & + & \\
\hline & 32 & + & ++ & \\
\hline & 33 & + & ++ & \\
\hline & 36 & + & & \\
\hline $\mathrm{C}_{6}-\mathrm{C}_{1}$ type & 3 & ++ & ++ & ++++ \\
\hline \multirow[t]{2}{*}{$\mathrm{C}_{6}-\mathrm{C}_{2}$ type } & 4 & + & & ++ \\
\hline & 37 & ++ & & \\
\hline \multirow[t]{3}{*}{$\mathrm{C}_{6}-\mathrm{C}_{3}$ type } & 5 & & ++ & + \\
\hline & 1 & & & ++++++ \\
\hline & 6 & & & + \\
\hline \multirow{2}{*}{ Acetylene glycoside } & 34 & & + & \\
\hline & 2 & & & + \\
\hline Alkyl glycoside & 30 & & + & \\
\hline \multirow{2}{*}{ Amino acids } & 38 & + & & \\
\hline & 10 & & ++++ & ++ \\
\hline
\end{tabular}

$1-9 \mathrm{mg},+; 10-19 \mathrm{mg},++; 20-39 \mathrm{mg},+++; 40-89 \mathrm{mg},++++; 90-199 \mathrm{mg},+++++; \geqq 200 \mathrm{mg},++++++$ (from $1.4 \mathrm{~kg}$ of $A$. japonica, $1.5 \mathrm{~kg}$ of $A$. lancea, and $1.5 \mathrm{~kg}$ of $A$. ovata). 11: $(1 S, 4 S, 5 S, 7 R, 10 R)-10,11,14$-trihydroxyguai-3-one $11-O$ - $\beta$-D-glucopyranoside, 12: $(1 S, 4 S, 5 R, 7 R, 10 R)$ - $10,11,14$-trihydroxyguai-3-one $11-O$ - $\beta$-D-glucopyranoside, 13: $(1 S, 5 R, 7 R, 10 R)$-secoatractylolactone 11- $O$ - $\beta$-D-glucopyranoside, 14: atractyloside A 14- $O$ - $\beta$-D-fructofuranoside, 15: $(1 S, 4 S, 5 S, 7 R, 10 S)$ - $10,11,14$-trihydroxyguai-3-one 11- $O$ - $\beta$-D-glucopyranoside, 16: $(5 R, 7 R, 10 S)$-isopterocarpolone $\beta$-D-glucopyranoside, 17: atractyloside I, 18: cis-atractyloside I, 19: atractyloside C, 20: atractyloside D, 21: atractyloside E, 22: atractyloside G, 23: $(2 R, 3 R, 5 R, 7 R, 10 S)$-atractyloside G 2- $O$ - $\beta$-D-glucopyranoside, 24: $(1 R, 2 R, 4 S)$-2-hydroxy-1,8-cineole $\beta$-D-glucopyranoside, 25: $(1 S, 2 S, 4 R)$-2-hydroxy-1,8-cineole $\beta$-D-glucopyranoside, 26: (4S)- $p$-menth-1-ene-7,8-diol 8- $O$ - $\beta$-D-glucopyranoside, 27: $(1 S, 2 R, 4 S)$ - $p$-menthane-1,2,8-triol 8- $O$ - $\beta$-D-glucopyranoside, 28: 3-methyl3-butenyl $\beta$-D-apiofuranosyl-( $1 \rightarrow 6$ )- $\beta$-D-glucopyranoside, 29: 3 -methyl-2-butenyl $\beta$-D-apiofuranosyl-( $1 \rightarrow 6)-\beta$-D-gluco-pyranoside, 30: isopropyl $\beta$-D-apiofuranosyl-( $1 \rightarrow 6$ )- $\beta$-Dglucopyranoside, 31: 4-hydroxy-3-methoxyphenyl $\beta$-D-glucopyranoside, 32: 4-hydroxy-3-methoxyphenyl $\beta$-D-apiofuranosyl-(1 $\rightarrow 6$ )- $\beta$-D-glucopyranoside, 33: 4-hydroxy-3methoxyphenyl $\beta$-D-xylopyranosyl-(1 $\rightarrow 6)-\beta$-D-glucopyranoside, 34: $(2 E, 8 E)$-2,8-decadiene-4,6-diyne-1,10-diol $11-O$ - $\beta$-D-glucopyranoside, 35: (3R,5S,8S,10S)-3-hydroxyatractylerolide III 3-O- $\beta$-D-glucopyranoside, 36: seguinoside B, 37: phenethyl $\alpha$-L-rhamnopyranosyl-( $1 \rightarrow 6)-\beta$-D-glucopyranoside, 38: L-phenyalanine.

${ }^{13} \mathrm{C}-\mathrm{NMR}\left(\mathrm{DMSO}-d_{6}, 67.5 \mathrm{MHz}\right) \delta: 160.72(\mathrm{C}-2), 111.51(\mathrm{C}-3), 144.52(\mathrm{C}-$ 4), $109.56(\mathrm{C}-5), 145.36(\mathrm{C}-6), 151.49(\mathrm{C}-7), 102.77$ (C-8), $149.61(\mathrm{C}-9)$, $110.40(\mathrm{C}-10), 56.01\left(6-\mathrm{OCH}_{3}\right)$.

(2E)-2-Decene-4,6-diyne-1,8-diol 8-O- $\beta$-D-Apiofuranosyl-( $1 \rightarrow 6)-\beta$-Dglucopyranoside (2): An amorphous powder, $[\alpha]_{\mathrm{D}}^{23}-144^{\circ}(c=0.1, \mathrm{MeOH})$. Positive FAB-MS $m / z$ : $459.1870[\mathrm{M}+\mathrm{H}]^{+}$(Calcd for $\mathrm{C}_{21} \mathrm{H}_{31} \mathrm{O}_{11}, 459.1867$ ), $183\left[\mathrm{M}-\mathrm{C}_{11} \mathrm{H}_{20} \mathrm{O}_{10}+\mathrm{H}\right]^{+}$(base). Negative FAB-MS m/z: $457[\mathrm{M}-\mathrm{H}]^{-}, 181$ $\left[\mathrm{M}-\mathrm{C}_{11} \mathrm{H}_{20} \mathrm{O}_{10}-\mathrm{H}\right]^{-}$(base). ${ }^{1} \mathrm{H}-\mathrm{NMR}$ (pyridine- $\left.d_{5}, 500 \mathrm{MHz}\right) \delta: 4.41(2 \mathrm{H}$, br s, $\left.\mathrm{H}_{2}-1\right), 6.67(1 \mathrm{H}, \mathrm{td}, J=4.0,16.0 \mathrm{~Hz}, \mathrm{H}-2), 6.25(1 \mathrm{H}, \mathrm{d}, J=16.0 \mathrm{~Hz}, \mathrm{H}-$ 3), $5.11(1 \mathrm{H}, \mathrm{dd}, J=6.5,6.5 \mathrm{~Hz}, \mathrm{H}-8), 1.84\left(2 \mathrm{H}, \mathrm{m}, \mathrm{H}_{2}-9\right), 0.99(3 \mathrm{H}, \mathrm{t}$, $\left.J=7.5 \mathrm{~Hz}, \mathrm{H}_{3}-10\right), 5.25(1 \mathrm{H}, \mathrm{d}, J=7.5 \mathrm{~Hz}, \mathrm{Glc} \mathrm{H}-1), 5.81(1 \mathrm{H}, \mathrm{d}, J=2.5 \mathrm{~Hz}$, Api H-1). ${ }^{13} \mathrm{C}-\mathrm{NMR}$ (pyridine- $\left.d_{5}, 125 \mathrm{MHz}\right) \delta: 61.89$ (C-1), 149.91 (C-2), 106.92 (C-3), 77.92 (C-4), 74.33 (C-5), 71.20 (C-6), 82.26 (C-7), 69.14 (C8), 29.34 (C-9), 9.76 (C-10), 101.91 (Glc C-1), 75.02 (Glc C-2), 78.53 (Glc C-3), 71.79 (Glc C-4), 77.34 (Glc C-5), 68.89 (Glc C-6), 111.31 (Api C-1), 77.80 (Api C-2), 80.45 (Api C-3), 75.03 (Api C-4), 65.57 (Api C-5). HMBC correlations: H-2/C-1, C-3,C-4; H-3/C-5; H-8/C-6, C-7, C-9, C-10, Glc C-1;

\section{H2-9/C-7, C-8, C-10; H3-10/C-8, C-9; Glc H-1/C-8; Api H-1/Glc C-6.}

\section{References}

1) Kitajima J., Kamoshita A., Ishikawa T., Takano A., Fukuda T., Isoda S., Ida Y., Chem. Pharm. Bull., 51, 152-157 (2003).

2) Yahara S., Higashi T., Iwaki K., Nohara T., Marubayashi N., Ueda I., Kohoda H., Goto K., Izumi H., Nuno M., Katsuki S., Isoda S., Satake M., Chem. Pharm. Bull., 37, 2995-3000 (1989).

3) Kitajima J., Kamoshita A., Ishikawa T., Takano A., Fukuda T., Isoda S., Ida Y., Chem. Pharm. Bull., 51, 673-678 (2003).

4) "Japanese Pharmacopoeia," 14th edition, Hirokawa Publishing Co., Tokyo, 2001, pp. 2708-2709, 2869-2871.

5) Kohjyouma M., Kohda H., Biol. Pharm. Bull., 20, 502-506 (1997).

6) Tsukamoto H., Hisada S., Nishibe S., Chem. Pharm. Bull., 33, 396399 (1985).

7) Kitajima J., Ishikawa T., Tanaka Y., Ono M., Ito Y., Nohara T., Chem. Pharm. Bull., 46, 1587-1590 (1998).

8) Kisiel W., Braszcz B., Fitoterapia, 71, 269-273 (2000). 